\title{
EXPONENTIAL BASES ON TWO DIMENSIONAL TRAPEZOIDS
}

\author{
LAURA DE CARLI AND ANUDEEP KUMAR
}

(Communicated by Alexander Iosevich)

\begin{abstract}
We discuss the existence and stability of Riesz bases of exponential type of $L^{2}(T)$ for special domains $T \subset \mathbb{R}^{2}$ called trapezoids, and we construct exponential bases on the finite union of rectangles with the same height. We also generalize our main theorems in dimension $d \geq 3$.
\end{abstract}

\section{INTRODUCTION}

The study of Riesz bases in Hilbert spaces has been a fruitful topic of investigation for many decades. Riesz bases allow us to decompose $L^{2}$ functions (which can be thought of as signals) in a unique way into a sum of basic frequencies. Riesz bases of exponential type, i.e., in the form of $\mathcal{B}=\left\{e^{i \alpha_{n}(x)}\right\}_{n \in \mathbb{Z}}$, with $\alpha_{n} \in L^{\infty}(D)$, are especially simple and easy to work with. Here, $D$ is a domain of $\mathbb{R}^{d}$, i.e., a bounded and measurable set of finite Lebesgue measure. If the $\alpha_{n}(x)$ 's are linear, $\mathcal{B}$ is called an exponential basis. Exponential bases of $L^{2}(a, b)$ have been well studied since Paley and Wiener [17] and Levinson [12] explored the possibility of non-harmonic Fourier series (see also [3], 7]). Since then, exponential bases have been used in various fields of mathematics such as the theory of non-selfadjoint operators, control theory and signal processing. See [2, [4, [8, just to cite a few. Much less is known about the existence of exponential bases of $L^{2}(D)$ when $D$ has dimension $d \geq 2$. In [15] the existence of exponential bases is proved for a convex symmetric polygon. To the best of our knowledge, the only results in the literature in dimension greater than two are in [16] and in the very recent 9 .

Exponential bases in dimension $d=2$ and $d=3$ are important for the applications to image recognition, magnetic resonance imaging and data compression. We refer the interested reader to the recent [6] and the references cited there.

In this paper we construct Riesz bases of exponential type on a class of domains of $\mathbb{R}^{2}$ that, with some abuse of terminology, are called trapezoids. Let $-\infty<a<$ $b<\infty$, and let $f:[a, b] \rightarrow \mathbb{R}$. Assume that

$$
0<\ell<f(x)<M \quad \text { almost everywhere (a.e) on }[a, b] \text {. }
$$

We refer to the set

$$
T=\left\{(x, y) \in \mathbb{R}^{2}:|x| \leq f(y), a \leq y \leq b\right\}
$$

as the trapezoid bounded by $f$.

In what follows we will assume that trapezoids are bounded by functions that satisfy (1.1), and we will let $[a, b]=[0,1]$ for the sake of simplicity.

Received by the editors September 24, 2012 and, in revised form, June 19, 2013.

2010 Mathematics Subject Classification. Primary 42C15, 42C30.

Key words and phrases. Exponential bases, trapezoids, multi-rectangles. 
A trapezoid bounded by a step function is a union of a finite family of rectangles. These trapezoids are especially interesting because step functions are dense in $L^{p}(0,1)$ for every $p<\infty$. We say that a positive step function is regular if it is constant on intervals of equal length; thus, a regular step function on $[0,1]$ can be written as

$$
s(x)=\sum_{j=1}^{N-1} b_{j} \chi_{\left[\frac{j-1}{N}, \frac{j}{N}\right)}(x)+b_{N} \chi_{\left[\frac{N-1}{N}, 1\right]}(x)
$$

where $\chi$ is the characteristic function, $N$ a positive integer and $b_{j}>0$. A trapezoid bounded by a step function as in (1.3) is called a multi-rectangle with $N$ steps.

In Section 3 we approximate the characteristic function of a trapezoid with regular step functions to construct special Riesz bases of exponential type on $L^{2}(T)$.

Theorem 1.1. Let $T$ be the trapezoid bounded by a piecewise continuous $f$. There exists a family of regular step functions $\left\{s_{n}\right\}_{n \geq 1}$ which converge uniformly to $f$, for which

$$
\mathcal{B}=\left\{e^{\pi i\left(\frac{n x}{s_{n}(y)}+2 k y\right)}\right\}_{n, k \in \mathbb{Z}}
$$

is a Riesz basis of $L^{2}(T)$.

Recall that $f$ is piecewise continuous if it is continuous everywhere except at a finite number of points. Theorem 1.1 allows us to construct a Riesz basis of $L^{2}(T)$ in the form of $\left\{e^{i\left(x \lambda_{n}(y)+2 k y\right)}\right\}_{n, k \in \mathbb{Z}}$, with $\lambda_{n}(y)=n / s_{n}(y)$ piecewise constant. If $\lambda_{n}$ was constant, then $\mathcal{B}$ would be an exponential basis of $L^{2}(T)$, but the piecewise constant functions are the "next best thing" after the constants.

Theorem 1.1 is a corollary of the following

Theorem 1.2. Let $T$ be the trapezoid bounded by $f$. Let $\left\{g_{n}(y)\right\}_{n \in \mathbb{Z}} \subset L^{\infty}(0,1)$ be such that

$$
\left|\frac{f(y)}{g_{n}(y)}-1\right|=\epsilon_{n}(y)<\frac{1}{4|n|} \quad \text { a.e. }
$$

Then, $\mathcal{B}=\left\{e^{\pi i\left(\frac{n x}{g_{n}(y)}+2 k y\right)}\right\}_{n, k \in \mathbb{Z}}$ is a Riesz basis for $L^{2}(T)$. The constant $\frac{1}{4}$ in (1.5) cannot be replaced by any larger constant.

This theorem can be viewed as an analogue of the celebrated Kadec stability theorem that we state and discuss in Section 2.3.

In Section 4 we show that trapezoids bounded by regular step functions have exponential bases. We prove the following

Theorem 1.3. Let $R \subset[-1,1] \times[0,1]$ be a multi-rectangle with $N$ steps. There exists sequences $\left\{n_{k}\right\}_{k \in \mathbb{Z}},\left\{m_{h}\right\}_{h \in \mathbb{Z}} \subset \mathbb{Z}$ such that $\left\{e^{2 \pi i\left(\frac{n_{k} x}{2 N}+N m_{h} y\right)}\right\}_{h, k \in \mathbb{Z}}$ is a Riesz basis of $L^{2}(R)$.

To prove Theorem 1.3 we show that an exponential basis of $L^{2}(R)$ can be obtained from an exponential basis on a finite union of disjoint segments of $\mathbb{R}$ (or multi-intervals). It has been recently proved by G. Kozma and S. Nitzan that every multi-interval has an exponential basis. The proof of Theorem 1.3 relies on the main theorem in 11 .

Interest in exponential Riesz bases on multi-intervals has its roots in practical applications for the sampling of band-limited signals, and the first partial results 
came from there. The main theorem in [11] improves results of N. Lev [12] and K. Seip [19].

The plan of this paper is the following. In Section 2 we state some preliminary definitions and results. In Section 3 we prove Theorems 1.1 and 1.2. In Section 4 we prove Theorem 1.3. In Section 5 we prove analogues of our main theorems in dimensions $d \geq 2$. We have stated some remarks and conjectures in Section 6 .

\section{Preliminaries}

In this section we have collected some definitions and preliminary results. We refer to the textbooks [22] and to the excellent paper [5] for a survey on bases and frames in Hilbert spaces.

2.1. Frames and Riesz bases. A sequence of vectors $\mathcal{B}=\left\{v_{1}, v_{2}, v_{3}, \ldots\right\}$ in a Hilbert space $H$ is a frame if there exist constants $A, B>0$ such that for every $w \in H$,

$$
A\|w\|^{2} \leq \sum_{j=1}^{\infty}\left|\left\langle w, v_{j}\right\rangle\right|^{2} \leq B\|w\|^{2} .
$$

Here, \|\|$=\|\|_{H}$ and \langle\rangle$=\langle\rangle_{H}$ are the norm and the inner product in $H$. $\mathcal{B}$ is a tight frame if $A=B$.

We say that $\mathcal{B}$ is a Riesz basis if it is the image of an orthonormal basis through a linear, bounded and invertible operator on $H$. Equivalently, $\mathcal{B}$ is a Riesz basis if it is an exact frame, i.e, if it ceases to be a frame when any of its elements is removed. See e.g. 22 for other equivalent characterizations of Riesz basis.

Frames are over-complete Riesz basis and provide robust, basis-like representations of vectors in a Hilbert space. Because frames are not necessarily linearly independent, they are often more easily constructible than bases. For example, assume that $D \subset P=[-\pi, \pi] \times[-\pi, \pi]$. An exponential basis of $L^{2}(P)$ is $\mathcal{B}=\left\{e^{i(n x+m y)}\right\}_{n, m \in \mathbb{Z}}$. Let $f \in L^{2}(D)$, and let

$$
\tilde{f}(x)= \begin{cases}f(x) & \text { if } x \in D, \\ 0 & \text { if } x \in P-D .\end{cases}
$$

By the Plancherel identity, $\sum_{n, m \in \mathbb{Z}}\left\langle\tilde{f}, e^{i(n x+m y)}\right\rangle_{L^{2}(P)}^{2}=4 \pi^{2}\|\tilde{f}\|_{L^{2}(P)}^{2}$. Clearly, also $\sum_{n, m \in \mathbb{Z}}\left\langle f, e^{i(n x+m y)}\right\rangle_{L^{2}(D)}^{2}=4 \pi^{2}\|f\|_{L^{2}(D)}^{2}$, and so $\mathcal{B}_{\left.\right|_{D}}$, the restrictions of the functions of $\mathcal{B}$ to $D$, is a tight frame of $L^{2}(D)$.

The argument that we have used in this example proves the following

Proposition 2.1. Let $P$ be a domain in $\mathbb{R}^{d}$, and let $\mathcal{F}=\left\{v_{n}\right\}_{n \in \mathbb{Z}}$ be a frame on $L^{2}(P)$. For every domain $D \subset P$, the set $\mathcal{F}_{\left.\right|_{D}}$ is a frame on $L^{2}(D)$.

The previous example shows that we can always construct exponential frames on $L^{2}(D)$, but it is often difficult, and sometimes impossible, to extract exponential bases from them. That may depend on the shape of $D$, but also on the frame itself. K. Seip proved in [18] that there are exponential frames of $L^{2}(-\pi, \pi)$ which do not contain an exponential basis. However, it is proved in [19] that for every $L>1$, the set $\mathcal{B}=\left\{e^{i \pi x \frac{n}{L}}\right\}_{n \in \mathbb{Z}}$ (which is a frame of $L^{2}(-1,1)$ by Proposition 2.1) contains an exponential basis of $L^{2}(-1,1)$; that is, there exists a sequence of integers $\left\{n_{k}\right\}_{k \in \mathbb{Z}}$ such that $\mathcal{B}^{\prime}=\left\{e^{i \pi x \frac{n_{k}}{L}}\right\}_{k \in \mathbb{Z}}$ is an exponential basis of $L^{2}(-1,1)$. 
The main theorem in 11 shows that $\mathcal{B}$ contains a Riesz basis of $L^{2}(I)$ also when $I \subset(-L, L)$ is a multi-interval.

Theorem 2.2 (Kozma and Nitzan). Let $I \subset \mathbb{R}$ be a finite union of intervals. Then there exists a sequence $\left\{\lambda_{k}\right\}_{k \in \mathbb{Z}} \subset \mathbb{R}$ such that the functions $\left\{e^{2 \pi i \lambda_{k} x}\right\}_{k \in \mathbb{Z}}$ form a Riesz basis in $L^{2}(I)$. Moreover, if $I \subset[-L, L]$, then we can choose $\lambda_{k}=\frac{n_{k}}{2 L}$, with $n_{k} \in \mathbb{Z}$.

2.2. Stability of Riesz bases. Riesz bases are stable, in the sense that a small perturbation of a Riesz basis produces a Riesz basis. Let us recall the Paley-Wiener stability theorem, and the celebrated Kadec $\frac{1}{4}$-theorem for exponential bases of $L^{2}(-a, a)$. The proof of both theorems can be found e.g. in [22]. The Kadec theorem was originally proved in [14].

Theorem 2.3 (Paley-Wiener). Let $\left\{v_{n}\right\}_{n \in \mathbb{N}}$ be a Riesz basis for a Hilbert space $H$. Suppose that $\left\{w_{n}\right\}_{n \in \mathbb{N}}$ is a sequence of elements of $H$ such that

$$
\left\|\sum_{j=1}^{n} c_{j}\left(v_{j}-w_{j}\right)\right\| \leq \lambda\left\|\sum_{j=1}^{n} c_{j} v_{j}\right\|
$$

for some constant $0<\lambda<1$ and all choices of scalars $c_{1}, \ldots, c_{n}$. Then $\left\{w_{n}\right\}_{n \in \mathbb{N}}$ is a Riesz basis for $H$.

Note that if $\left\{v_{n}\right\}_{n \in \mathbb{N}}$ is an orthonormal basis of $H$, and $\sum_{j=1}^{n} c_{j}^{2}=1$, then the right hand side of (2.2) equals $\lambda$.

Theorem 2.4 (Kadec). Let $a>0$, and let $\left\{\alpha_{n}\right\}_{n \in \mathbb{Z}} \subset \mathbb{R}$ be such that

$$
\left|\frac{a}{\alpha_{n}}-1\right|=\epsilon_{n}<\frac{1}{4|n|} \text {. }
$$

Then, $\mathcal{B}=\left\{e^{i \pi \frac{n}{\alpha_{n}} x}\right\}_{n \in \mathbb{Z}}$ is a Riesz basis for $L^{2}(-a, a)$. The constant $\frac{1}{4}$ cannot be replaced by any larger number.

The Kadec theorem has been extensively celebrated and generalized (see e.g. [1], 21]). Theorem 1.2 is a generalization of Theorem 2.4. Indeed, the inequality (1.5) reduces to (2.3) if $f(y) \equiv a$ (i.e, if $T$ is a rectangle) and $g_{n}(y) \equiv \alpha_{n}$. See also Theorem 1.1 in 20$]$.

\section{BASES OF EXPONENTIALS OF $L^{2}$ OF TRAPEZOIDS}

In this section we prove Theorem 1.2 and its corollary, Theorem 1.1. We start with an easy construction of a Riesz basis for $L^{2}(T)$.

Lemma 3.1. Let $T$ be the trapezoid bounded by $f$. Then,

$$
\mathcal{B}=\left\{e^{\pi i\left(\frac{n x}{f(y)}+2 k y\right)}\right\}_{n, k \in \mathbb{Z}}
$$

is a Riesz basis on $L^{2}(T)$.

Proof. Recall that bounded, linear and invertible functions between Hilbert spaces maps Riesz bases into Riesz bases. We let $R=[-1,1] \times[0,1]$, and $\mathcal{L}: L^{2}(R) \rightarrow$ $L^{2}(T)$ be such that

$$
\mathcal{L}(\psi)(x, y)=(f(y))^{-\frac{1}{2}} \psi(x / f(y), y) .
$$


$\mathcal{L}$ is an invertible isometry. Since $\mathcal{C}=\left\{\frac{1}{\sqrt{2}} e^{\pi i(n x+2 k y)}\right\}_{n, k \in \mathbb{Z}}$ is an orthonormal basis of $L^{2}(R)$, the set $\mathcal{B}_{1}=\mathcal{L}(\mathcal{C})=\left\{(2 f(y))^{-\frac{1}{2}} e^{\pi i\left(\frac{n x}{f(y)}+2 k y\right)}\right\}_{n, k \in \mathbb{Z}}$ is an orthonormal basis for $L^{2}(T)$.

The map $G(\psi)(x, y)=(2 f(y))^{\frac{1}{2}} \psi(x, y)$ is linear and invertible on $L^{2}(T)$ because $f(y)>\ell>0$, and so $\mathcal{B}$, the image of $\mathcal{B}_{1}$ through $G$, is a Riesz basis for $L^{2}(T)$.

Proof of Theorem 1.2. By Lemma 3.1, $\mathcal{B}_{1}=\left\{(2 f(y))^{-\frac{1}{2}} e^{\pi i\left(\frac{n x}{f(y)}+2 k y\right)}\right\}_{n, k \in \mathbb{Z}}$ is an orthonormal Riesz basis for $L^{2}(T)$. Let $\left\{g_{n}\right\}_{n \in \mathbb{Z}} \subset L^{\infty}(0,1)$ be as in (1.5). Let $\tilde{\mathcal{B}}=\left\{(2 f(y))^{-\frac{1}{2}} e^{\pi i\left(\frac{n x}{g_{n}(y)}+2 k y\right)}\right\}_{n, k \in \mathbb{Z}}$. We show that

$$
\int_{T}\left|\sum_{n, k \in \mathbb{Z}} \frac{c_{n, k}}{(2 f(y))^{\frac{1}{2}}}\left(e^{\pi i\left(\frac{n x}{f(y)}+2 k y\right)}-e^{\pi i\left(\frac{n x}{g_{n}(y)}+2 k y\right)}\right)\right|^{2} d x d y \leq \lambda^{2}<1
$$

whenever $\sum_{n, k \in \mathbb{Z}}\left|c_{n, k}\right|^{2} \leq 1$. By the Paley-Wiener theorem (Theorem 2.3 ), $\tilde{\mathcal{B}}$ is a Riesz basis of $L^{2}(T)$, and so also $\mathcal{B}=\left\{e^{\pi i\left(\frac{n x}{g_{n}(y)}+2 k y\right)}\right\}_{n, k \in \mathbb{Z}}$ is a Riesz basis for $L^{2}(T)$.

In order to simplify the proof of (3.3), we use the change of variables $(x, y) \rightarrow$ $(x f(y), y)$ in the integral. With this change of variables, $T$ is mapped into $R=$ $[-1,1] \times[0,1]$, and $(3.3)$ reduces to

$$
\left\|\sum_{n, k \in \mathbb{Z}} \frac{c_{n, k}}{\sqrt{2}}\left(e^{\pi i(n x+2 k y)}-e^{\pi i\left(\frac{n x f(y)}{g_{n}(y)}+2 k y\right)}\right)\right\|_{L^{2}(R)} \leq \lambda<1 .
$$

In the rest of the proof, \|\|$=\|\|_{L^{2}(R)}$. We argue as in the proof of the Kadec theorem. We let

$$
e^{\pi i(n x+2 k y)}-e^{\pi i\left(\frac{n x f(y)}{g_{n}(y)}+2 k y\right)}=e^{\pi i(n x+2 k y)}\left(1-e^{i \delta_{n} x}\right)
$$

where $\delta_{n}=\delta_{n}(y)=\pi n\left(\frac{f(y)}{g_{n}(y)}-1\right)$. By (1.5)

$$
L=\sup _{\substack{y \in[0,1] \\ n \in \mathbb{Z}}}\left|\delta_{n}\right|=\sup _{n \in \mathbb{Z}}\left(\pi|n| \sup _{y \in[0,1]}\left|\frac{f(y)}{g_{n}(y)}-1\right|\right)<\frac{\pi}{4} .
$$

We expand the function $1-e^{i \delta_{n} x}$ in $L^{2}(-1,1)$ as a Fourier series relative to the complete orthonormal system $\left\{1, \cos (x \pi n), \sin \left(\left(n-\frac{1}{2}\right) \pi x\right)\right\}_{n=1}^{\infty}$. We obtain

$$
\begin{gathered}
1-e^{i \delta_{n} x}=\left(1-\frac{\sin \delta_{n}}{\delta_{n}}\right)+2 \sum_{m=1}^{\infty} \frac{(-1)^{m} \delta_{n} \sin \delta_{n}}{m^{2} \pi^{2}-\delta_{n}^{2}} \cos (\pi m x) \\
+2 i \sum_{m=1}^{\infty} \frac{(-1)^{m} \delta_{n} \cos \delta_{n}}{\left(m-\frac{1}{2}\right)^{2} \pi^{2}-\delta_{n}^{2}} \sin \left(\left(m-\frac{1}{2}\right) \pi x\right) .
\end{gathered}
$$


To estimate the $L^{2}$ norm in (3.3), we use the above Fourier series, change the order of the summation and use the triangle inequality; thus,

$$
\begin{aligned}
& \left\|\sum_{n, k \in \mathbb{Z}} \frac{c_{n, k}}{\sqrt{2}} e^{\pi i(n x+2 k y)}\left(1-e^{i \delta_{n} x}\right)\right\| \\
\leq & \frac{1}{\sqrt{2}}\left\|\sum_{n, k \in \mathbb{Z}}\left(1-\frac{\sin \delta_{n}}{\delta_{n}}\right) c_{n, k} e^{\pi i(n x+2 k y)}\right\| \\
+ & \sqrt{2} \sum_{m=1}^{\infty}\left\|\cos (m \pi x) \sum_{n, k \in \mathbb{Z}} \frac{(-1)^{m} \delta_{n} \sin \delta_{n}}{m^{2} \pi^{2}-\delta_{n}^{2}} c_{n, k} e^{\pi i(n x+2 k y)}\right\| \\
+ & \sqrt{2} \sum_{m=1}^{\infty}\left\|\sin \left(\left(m-\frac{1}{2}\right) \pi x\right) \sum_{n, k \in \mathbb{Z}} \frac{(-1)^{m} \delta_{n} \cos \delta_{n}}{\left(m-\frac{1}{2}\right)^{2} \pi^{2}-\delta_{n}^{2}} c_{n, k} e^{\pi i(n x+2 k y)}\right\| \\
= & A+B+C .
\end{aligned}
$$

(3.4) follows if we prove that $A+B+C<1$; to estimate $A$, we use the Plancherel theorem,

$$
\begin{aligned}
& A^{2}=\frac{1}{2} \int_{0}^{1} \int_{-1}^{1}\left|\sum_{k \in \mathbb{Z}} \sum_{n \in \mathbb{Z}}\left(1-\frac{\sin \delta_{n}}{\delta_{n}}\right) c_{n, k} e^{i n \pi x+2 \pi i k y}\right|^{2} d x d y \\
& =\frac{1}{2} \int_{0}^{1}\left(\int_{-1}^{1}\left|\sum_{n \in \mathbb{Z}}\left(1-\frac{\sin \delta_{n}}{\delta_{n}}\right)\left(\sum_{k \in \mathbb{Z}} c_{n, k} e^{2 \pi i k y}\right) e^{i n \pi x}\right|^{2} d x\right) d y \\
& =\int_{0}^{1} \sum_{n \in \mathbb{Z}}\left(1-\frac{\sin \delta_{n}}{\delta_{n}}\right)^{2}\left|\sum_{k \in \mathbb{Z}} c_{n, k} e^{2 \pi i k y}\right|^{2} d y,
\end{aligned}
$$

and since the function $t \rightarrow 1-\frac{\sin t}{t}$ is increasing when $t \in[0, \pi]$, and $\left|\delta_{n}\right|<L<\frac{\pi}{4}$ by (3.5), we obtain

$$
\begin{aligned}
A^{2} & \leq\left(1-\frac{\sin L}{L}\right)^{2} \sum_{n \in \mathbb{Z}} \int_{0}^{1}\left|\sum_{k \in \mathbb{Z}} c_{n, k} e^{2 \pi i k y}\right|^{2} d y \\
& =\left(1-\frac{\sin L}{L}\right)^{2} \sum_{k \in \mathbb{Z}} c_{n, k}^{2}=\left(1-\frac{\sin L}{L}\right)^{2} .
\end{aligned}
$$

We argue in a similar way to show that

$$
B \leq \sum_{m=1}^{\infty} \frac{2 L \sin L}{\pi^{2} m^{2}-L^{2}} \leq \sum_{m=1}^{\infty} \frac{2 L \cos L}{\pi^{2}\left(m-\frac{1}{2}\right)^{2}-L^{2}} .
$$

Thus,

$$
A+B+C \leq 1-\frac{\sin L}{L}+\sum_{m=1}^{\infty} \frac{\frac{2 L}{\pi} \sin L}{\pi\left(m^{2}-\frac{L^{2}}{\pi^{2}}\right)}+\sum_{m=1}^{\infty} \frac{\frac{2 L}{\pi} \cos L}{\pi\left(\left(m-\frac{1}{2}\right)^{2}-\frac{L^{2}}{\pi^{2}}\right)} .
$$


The series $\sum_{m=1}^{\infty} \frac{\frac{2 L}{\pi}}{\pi\left(m^{2}-\frac{L^{2}}{\pi^{2}}\right)}$ and $\sum_{m=1}^{\infty} \frac{\frac{2 L}{\pi}}{\pi\left(\left(m-\frac{1}{2}\right)^{2}-\frac{L^{2}}{\pi^{2}}\right)}$ are the partial fraction expansions of the function $\frac{1}{L}-\cot L$ and $\tan L$, respectively. Hence, $\lambda=A+B+C=$ $1-\cos L+\sin L$. Since $L<\frac{\pi}{4}$, we have $\lambda<1$, and (3.4) is proved.

To show that the constant in (1.5) cannot be replaced by a smaller constant, we use a straightforward generalization of an example by Ingham. We let $g_{n}(y)=$ $\frac{n f(y)}{n-\frac{\operatorname{sgn}(\mathrm{n})}{4}}$ if $n \neq 0$, and $g_{0}(y)=0$. Here $\operatorname{sgn}(n)=\frac{n}{|n|}$. The $g_{n}$ 's satisfy (1.5) with $\delta_{n}=\frac{1}{4 n}$. Let $v_{n, k}(x, y)=e^{\pi i\left(\frac{n x}{g_{n}(y)}+2 k y\right)}$. If $\left\{v_{n, k}\right\}_{m \in \mathbb{Z}}$ was a Riesz basis of $L^{2}(T)$, then $\left\{v_{n, k}(x, \bar{y})\right\}_{n \in \mathbb{Z}}$ would be a Riesz basis of $L^{2}(-f(\bar{y}), f(\bar{y}))$ for a.e. $\bar{y} \in(0,1)$. By a change of variables, the set $\left\{e^{i \pi x\left(n-\frac{\operatorname{sgn}(\mathrm{n})}{4}\right)}\right\}_{n \in \mathbb{Z}}$ would be a Riesz basis of $L^{2}(-1,1)$, but in [10] it is proved that this is not the case.

Proof of Theorem 1.1. Assume that $f$ is continuous and $\sup _{y \in[0,1]}|f(y)|=1$ (the proof of the general case is very similar). We construct a family of step functions $\left\{s_{n}\right\}_{n \geq 1}$ that satisfy the assumptions of Theorem 1.2, since we have assumed $|f(y)| \leq 1,(1.5)$ follows if we prove that

$$
\sup _{y \in[0,1]}\left|\frac{1}{s_{n}(y)}-\frac{1}{f(y)}\right|<\frac{1}{4 n} .
$$

Since $f>\ell>0, \frac{1}{f(y)}$ is continuous, it is thus also uniformly continuous in $[0,1]$. If we let $\epsilon=\frac{1}{4 n}$, there exists $\eta=\eta(n)>0$ such that $\left|\frac{1}{f(z)}-\frac{1}{f(y)}\right|<\frac{1}{4 n}$ whenever $|z-y|<\eta$. We partition $[0,1]$ with $N=N(n)$ intervals of length $\frac{1}{N}<\eta$. For $1 \leq j \leq N$, we let $y_{j}=\frac{j-1}{N}$; we can see at once that

$$
\left|\frac{1}{f\left(\frac{j-1}{N}\right)}-\frac{1}{f(y)}\right|<\frac{1}{4 n} \quad \text { if } \quad \frac{j-1}{N}<y<\frac{j}{N} .
$$

We let $s_{n}(y) \equiv f\left(\frac{j-1}{N}\right)$ if $\frac{j-1}{N} \leq y<\frac{j}{N}$ for every $1 \leq j \leq N-1$, and $s_{n}(y) \equiv f\left(\frac{N-1}{N}\right)$ if $\frac{N-1}{N} \leq y \leq 1$. The $s_{n}$ 's are step functions that satisfy (3.6). Since

$$
\sup _{y \in[0,1]}\left|s_{n}(y)-f(y)\right|=\sup _{y \in[0,1]} s_{n}(y) f(y)\left|\frac{1}{s_{n}(y)}-\frac{1}{f(y)}\right|<\frac{1}{4 n},
$$

the $s_{n}$ 's converge uniformly to $f$, as required.

\section{Proof of Theorem 1.3}

Let $R \subset[-1,1] \times[0,1]$ be a multi-rectangle with $N$ steps in $\mathbb{R}^{2}$. We let $h=\frac{1}{N}$, and $R=\bigcup_{j=1}^{N} \overline{R_{j}}$, where $R_{j}=\left(-b_{j}, b_{j}\right) \times((j-1) h, j h)$. Recall that we have assumed $0<b_{j} \leq 1$; after a dilation, we can assume $h=1$.

To prove Theorem 1.3 we associate to $R$ a multi-interval $I$, and we define an isometry $\mathcal{L}: L^{2}(\bar{I} \times[0,1]) \rightarrow L^{2}(R)$; then we construct an exponential basis of $L^{2}(\bar{I} \times[0,1])$, and we show that it is mapped by $\mathcal{L}$ into an exponential basis of $L^{2}(R)$.

Let $\vec{v}_{j}=(2(j-1),-(j-1))$, so that

$$
\tau_{\vec{v}_{j}} R_{j}=\left(-b_{j}+2(j-1), b_{j}+2(j-1)\right) \times(0,1)
$$


and $\bigcup_{j=1}^{N} \tau_{\vec{v}_{j}} R_{j}=I \times(0,1)$, where we have let

$$
I=\bigcup_{j=1}^{N}\left(-b_{j}+2(j-1), b_{j}+2(j-1)\right) .
$$

Note that $I \subset\left(-b_{1}, 2 N-1\right) \subset(-1,2 N-1)$.

The segments in (4.1) do not overlap; indeed, for every $j \geq 1, b_{j}+2(j-1) \leq$ $-b_{j+1}+2 j \Longleftrightarrow b_{j}+b_{j+1} \leq 2$ which is true by assumption $b_{j} \leq 1$.

Let $\mathcal{L}: L^{2}(\bar{I} \times[0,1]) \rightarrow L^{2}(R)$

$$
\mathcal{L} \psi(x, y)=\sum_{j=1}^{N} \chi_{\bar{R}_{j}}(x, y) \psi \circ \tau_{\vec{v}_{j}}(x, y) .
$$

It is easy to verify that $\mathcal{L}$ is a linear invertible isometry, and hence it maps Riesz basis into Riesz basis.

Let $\mathcal{B}=\left\{e^{2 \pi i \lambda_{k} x}\right\}_{k \in \mathbb{Z}}$ be an exponential basis of $L^{2}(I)$. By Theorem 2.2, we can assume $\lambda_{k}=\frac{n_{k}}{2 N}$ where $n_{k} \in \mathbb{Z}$. For a fixed $k \in \mathbb{Z}$, we choose $\left\{m_{h}\right\}_{h \in \mathbb{Z}}=$ $\left\{m_{k(h)}\right\}_{h \in \mathbb{Z}} \subset \mathbb{Z}$ with the following properties: the sequence $\left\{e^{2 \pi i m_{h} y}\right\}_{h \in \mathbb{Z}}$ is a Riesz basis of $L^{2}(0,1)$, and

$$
\begin{aligned}
\mathcal{L}\left(e^{2 \pi i\left(y m_{h}+x \lambda_{k}\right)}\right) & =\sum_{j=1}^{N} \chi_{\bar{R}_{j}}(x, y) e^{2 \pi i\left(\lambda_{k}(x+2(j-1))+m_{h}(y-j+1)\right)} \\
& =\chi_{R}(x, y) e^{2 \pi i\left(x \lambda_{k}+y m_{h}\right)} .
\end{aligned}
$$

We have (4.2) if $2 \pi(j-1)\left(2 \lambda_{k}-m_{h}\right)=2 \pi(j-1)\left(\frac{n_{k}}{N}-m_{h}\right)$ is an integer multiple of $2 \pi$. If we let $m_{h}=\left\{n_{k}\right\}+h$, where $\left\{n_{k}\right\}$ is the remainder of the division of $n_{k}$ by $N$, the sequence $\left\{e^{2 \pi i m_{h} y}\right\}_{h \in \mathbb{Z}}=\left\{e^{2 \pi i y\left(h+\left\{n_{k}\right\}\right)}\right\}_{h \in \mathbb{Z}}$ is an exponential basis of $L^{2}(0,1)$, and (4.2) is satisfied. By Lemma 4.1 below, the set $\mathcal{B}_{1}=\left\{e^{\pi i\left(y m_{h}+x \lambda_{k}\right)}\right\}_{h, k \in \mathbb{Z}}$ is an exponential basis of $L^{2}(\bar{I} \times[0,1])$, and so $\mathcal{L}\left(\mathcal{B}_{1}\right)$ is an exponential basis of $L^{2}(R)$.

We are left to prove the following

Lemma 4.1. Let $D$ be a domain of $\mathbb{R}^{k}$ and $E$ a domain of $\mathbb{R}^{d}$. Let $\left\{v_{n}(x)\right\}_{n \in \mathbb{Z}}$ be a Riesz basis of $L^{2}(D)$; assume that for every $n \in \mathbb{Z}$, there exists a sequence $\{n(m)\}_{m \in \mathbb{Z}} \subset \mathbb{Z}$ and a Riesz basis $\left\{w_{n(m)}(y)\right\}_{m \in \mathbb{Z}}$ of $L^{2}(E)$. Then

$$
\mathcal{B}=\left\{v_{n}(x) w_{n(m)}(y)\right\}_{n, m \in \mathbb{Z}}
$$

is a Riesz basis of $L^{2}(D \times E)$.

Proof. Let $\left\{v_{n}^{\prime}(x)\right\}_{n \in \mathbb{Z}}$ and $\left.\left\{w_{n(m)}^{\prime}(y)\right\}_{m \in \mathbb{Z}}\right)$ be dual bases of $\left\{v_{n}(x)\right\}$ and $\left\{w_{n(m)}(y)\right\}$ (see e.g. [22 for a definition of dual basis). Let $f(x, y) \in L^{2}(D \times E)$. Then, for a.e. $y \in E$, the function $x \rightarrow f(x, y)$ is in $L^{2}(D)$, and

$$
f(x, y)=\sum_{n=-\infty}^{\infty}\left\langle f, v_{n}^{\prime}\right\rangle_{D} v_{n}(x) .
$$

Let $f_{n}(y)=\left\langle f, v_{n}^{\prime}\right\rangle_{D}$. It is easy to verify that $f_{n} \in L^{2}(E)$, and so

$$
f_{n}(y)=\sum_{m=-\infty}^{\infty}\left\langle f_{n}, w_{n(m)}^{\prime}\right\rangle_{E} w_{n(m)}(y) \text {. }
$$


By the Fubini theorem, $\left\langle f_{n}, w_{n(m)}^{\prime}\right\rangle_{E}=\left\langle\left\langle f, v_{n}^{\prime}\right\rangle_{D}, w_{n(m)}^{\prime}\right\rangle_{E}=\left\langle f, v_{n}^{\prime} w_{n(m)}^{\prime}\right\rangle_{D \times E}$; therefore,

$$
\begin{aligned}
f(x, y) & =\sum_{n=-\infty}^{\infty} f_{n}(y) v_{n}(x)=\sum_{n=-\infty}^{\infty}\left(\sum_{m=-\infty}^{\infty}\left\langle f_{n}, w_{n(m)}^{\prime}\right\rangle_{E} w_{n(m)}(y)\right) v_{n}(x) \\
& =\sum_{n, m=-\infty}^{\infty}\left\langle f, w_{n(m)}^{\prime} v_{n}^{\prime}\right\rangle_{D \times E} w_{n(m)}(y) v_{n}(x)
\end{aligned}
$$

which shows that $w_{n(m)}(y) v_{n}(x)$ is a Riesz basis of $L^{2}(D \times E)$ with dual basis $w_{n(m)}^{\prime}(y) v_{n}^{\prime}(x)$.

\section{SPHERICAL TRAPEZOIDS}

In this section we prove a multi-dimensional version of Lemma 3.1 and we discuss generalizations of our main theorems in dimension $d \geq 2$. We will write $x \in \mathbb{R}^{d}$ as $\left(x^{\prime}, y\right)$, with $y \in \mathbb{R}$ and $x^{\prime} \in \mathbb{R}^{d-1}$. Let $f:[0,1] \rightarrow \mathbb{R}$ be as in (1.1). The trapezoid with spherical basis bounded by $f$ is the set

$$
T=\left\{\left(x^{\prime}, y\right) \in \mathbb{R}^{d}:\left|x^{\prime}\right| \leq f(y), 0 \leq y \leq 1\right\} .
$$

We say that $T$ is a multi-cylinder if $f$ is a regular step function.

We let $L_{S}^{2}(T)$ be the set of $L^{2}(T)$ functions which are radial in $x^{\prime}$. When $d=2$, a multi-cylinder is a multi-rectangle and $L_{S}^{2}(T)$ is the set of all $L^{2}(T)$ functions that are even in $x^{\prime}$.

The following lemma reduces the construction of a Riesz basis of $L_{S}^{2}(T)$ to that of a Riesz basis of $L^{2}(R)$, where $R=[0,1] \times[0,1]$. It is the analogue of Lemma 3.1 .

Lemma 5.1. Let $T$ be the spherical trapezoid bounded by $f$. Then,

$$
\mathcal{B}=\left\{e^{2 \pi i\left(\frac{n\left|x^{\prime}\right|}{f(y)}+k y\right)}\right\}_{n, k \in \mathbb{Z}}
$$

is a Riesz basis of $L_{S}^{2}(T)$.

Proof. Let $\mathcal{L}: L^{2}(R) \rightarrow L_{S}^{2}(T)$ be such that

$$
\mathcal{L}(\psi)\left(x^{\prime}, y\right)=\left(\left|S^{d-2}\right| f(y)\right)^{-\frac{1}{2}} r^{-\frac{d-2}{2}} \psi(r / f(y), y)
$$

where $r=\left|x^{\prime}\right|$ and $\left|S^{d-2}\right|$ is the measure of the unit sphere in $\mathbb{R}^{d-1}$. When $d=2$, we let $\left|S^{0}\right|=1$. $\mathcal{L}$ is an invertible isometry. Since $\mathcal{C}=\left\{e^{2 \pi i(n r+k y)}\right\}_{n, k \in \mathbb{Z}}$ is an orthonormal basis of $L^{2}(R)$, the set

$$
\mathcal{B}_{1}=\mathcal{L}(\mathcal{C})=\left\{\left(\left|S^{d-2}\right| f(y)\right)^{-\frac{1}{2}} r^{-\frac{d-2}{2}} e^{2 \pi i\left(\frac{n r}{f(y)}+k y\right)}\right\}_{n, k \in \mathbb{Z}}
$$

is an orthonormal basis of $L_{S}^{2}(T)$. We conclude that $\mathcal{B}$ is a Riesz basis of $L_{S}^{2}(T)$ as in Lemma 3.1

We can prove versions of Theorems $1.1,1.2$ and 1.3 for spherical trapezoids. The analogue of Theorem 1.1 is

Theorem 5.2. Let $T \subset \mathbb{R}^{d}$ be the spherical trapezoid bounded by a piecewise continuous $f$. There exists a family of regular step functions $\left\{s_{n}\right\}_{n \geq 1}$ which converge uniformly to $f$, for which $\mathcal{B}=\left\{e^{2 \pi i\left(\frac{n\left|x^{\prime}\right|}{s_{n}(y)}+k y\right)}\right\}_{n, k \in \mathbb{Z}}$ is a Riesz basis of $L_{S}^{2}(T)$. 
Theorem 1.1 is not a special case of Theorem 5.2 . indeed, when $d=2$, the set $\mathcal{B}$ defined above is not the same $\mathcal{B}$ as in Theorem 1.1. But the proof of Theorem 5.2 is almost a line-by-line repetition of the proof of Theorem 1.1, which follows from a straightforward generalization of Theorem 1.2 .

The analogue of Theorem 1.3 is

Theorem 5.3. Let $T \subset B_{1}(0) \times[0,1]$ be a multi-cylinder with $N$ steps. There exists sequences $\left\{n_{k}\right\}_{k \in \mathbb{Z}},\left\{m_{h}\right\}_{h \in \mathbb{Z}} \subset \mathbb{Z}$ such that $\left\{e^{2 \pi i\left(\frac{n_{k}\left|x^{\prime}\right|}{N}+N m_{h} y\right)}\right\}_{h, k \in \mathbb{Z}}$ is a Riesz basis of $L_{S}^{2}(T)$.

Our method of proof allows us to construct Riesz bases, but not exponential bases of $L_{S}^{2}(T)$. The existence of exponential bases on domains with radial symmetry is still an open problem.

\section{REMARKS AND OPEN PROBLEMS}

We have constructed exponential bases of $L^{2}(R)$ for certain multi-rectangles $R$; we are wondering if is it possible to do the same when $R$ is a union of a generic family of disjoint rectangles. In Theorem 1.3 the rectangles in $R$ have the same height, and this fact allows us to reduce the construction of an exponential basis of $L^{2}(R)$ to that of an exponential basis of $L^{2}(I)$. Our construction does not seem to work well for general multi-rectangles.

Complex analytic methods and the theory of Paley-Wiener spaces have often been used in these problems. We cite, for example, the recent paper by J. Marzo [16]; the author proves the existence of a Riesz basis of exponentials on a finite union of congruent cubes of $\mathbb{R}^{n}$ by finding complete interpolating sequences in a suitable Paley-Wiener space. We believe that the proof in [16] cannot be easily generalized when the cubes are replaced by parallelepiped.

We are also wondering if, for certain trapezoids $T$, the construction of exponential Riesz bases on a family of multi-rectangles $R_{n}$ that approximate $T$ (in the sense that the measure of the symmetric difference of $R_{n}$ and $T$ goes to zero when $n \rightarrow \infty$ ) can lead to the construction of an exponential basis of $L^{2}(T)$. We plan to pursue this investigation in a subsequent paper.

\section{ACKNOWLEDGEMENTS}

The authors wish to thank K. Seip for advice and suggestions, and the referee for suggesting multi-dimensional versions of our main theorems.

\section{REFERENCES}

[1] Avdonin, S. A. On the question of Riesz bases of exponential functions in $L^{2}$. (translated into English in: Vestnik Leningrad Univ. Ser. Mat., 13, pp 203-2011 (1979)

[2] S. A. Avdonin and S. A. Ivanov, Riesz bases of exponentials and divided differences (Russian, with Russian summary), Algebra i Analiz 13 (2001), no. 3, 1-17; English transl., St. Petersburg Math. J. 13 (2002), no. 3, 339-351. MR1850184 (2002g:42009)

[3] Richard Bellman, Convergence of non-harmonic Fourier series, Duke Math. J. 10 (1943), 551-552. MR0008431 (5,4b)

[4] Harold E. Benzinger, Nonharmonic Fourier series and spectral theory, Trans. Amer. Math. Soc. 299 (1987), no. 1, 245-259, DOI 10.2307/2000492. MR869410 (88m:42020)

[5] Peter G. Casazza, The art of frame theory, Taiwanese J. Math. 4 (2000), no. 2, 129-201. MR1757401 (2001f:42046)

[6] P. Casazza and G. Kutyniok, Finite Frames: Theory and Applications, Birkhauser, 2013. 
[7] R. J. Duffin and A. C. Schaeffer, A class of nonharmonic Fourier series, Trans. Amer. Math. Soc. 72 (1952), 341-366. MR0047179 (13,839a)

[8] Erich Freund and Joachim Petzold, Nonharmonic Fourier series: a formalism for analyzing signals (English, with French, German and Russian summaries), Elektron. Informationsverarb. Kybernet. 20 (1984), no. 10-11, 575-592. MR777071 (86m:94012)

[9] S. Grepstad and N. Lev, Multi-tiling and Riesz bases, arXiv:1212.4679 (2012)

[10] A. E. Ingham, Some trigonometrical inequalities with applications to the theory of series, Math. Z. 41 (1936), no. 1, 367-379, DOI 10.1007/BF01180426. MR1545625

[11] G. Kozma and S. Nitzan, Combining Riesz bases arXiv:1210.6383 (2012)

[12] Nir Lev, Riesz bases of exponentials on multiband spectra, Proc. Amer. Math. Soc. 140 (2012), no. 9, 3127-3132, DOI 10.1090/S0002-9939-2012-11138-4. MR2917085

[13] Norman Levinson, On non-harmonic Fourier series, Ann. of Math. (2) 37 (1936), no. 4, 919-936, DOI 10.2307/1968628. MR.1503319

[14] M. I. Kadec', The exact value of the Paley-Wiener constant (Russian), Dokl. Akad. Nauk SSSR 155 (1964), 1253-1254. MR0162088 (28 \#5289)

[15] Yurii I. Lyubarskii and Alexander Rashkovskii, Complete interpolating sequences for Fourier transforms supported by convex symmetric polygons, Ark. Mat. 38 (2000), no. 1, 139-170, DOI 10.1007/BF02384495. MR1749363 (2001m:32013)

[16] J. Marzo, Riesz basis of exponentials for a union of cubes in $\mathbb{R}^{d}$, arXiv:math/0601288 (2006)

[17] Raymond E. A. C. Paley and Norbert Wiener, Fourier transforms in the complex domain, American Mathematical Society Colloquium Publications, vol. 19, American Mathematical Society, Providence, RI, 1987. Reprint of the 1934 original. MR1451142 (98a:01023)

[18] Kristian Seip, On the connection between exponential bases and certain related sequences in $L^{2}(-\pi, \pi)$, J. Funct. Anal. 130 (1995), no. 1, 131-160, DOI 10.1006/jfan.1995.1066. MR 1331980 (96d:46030)

[19] Kristian Seip, A simple construction of exponential bases in $L^{2}$ of the union of several intervals, Proc. Edinburgh Math. Soc. (2) 38 (1995), no. 1, 171-177, DOI 10.1017/S0013091500006295. MR1317335 (96c:42006)

[20] Wenchang Sun and Xingwei Zhou, On the stability of multivariate trigonometric systems, J. Math. Anal. Appl. 235 (1999), no. 1, 159-167, DOI 10.1006/jmaa.1999.6386. MR1758675 (2001k:42040)

[21] Sun, W. and Zhou, X. On Kadec's $\frac{1}{4}-$ Theorem and the Stability of Gabor Frames. Applied and Comput. Harm. An. 7 (1999) no. 2, 239-242.

[22] Robert M. Young, An introduction to nonharmonic Fourier series, 1st ed., Academic Press Inc., San Diego, CA, 2001. MR1836633 (2002b:42001)

Department of Mathematics, Florida International University, Miami, Florida 33199

E-mail address: decarlil@fiu.edu

Department of Mathematics, The George Washington University, Washington, DC 20052

E-mail address: anudeep@email.gwu.edu 